\title{
Breast Cancer Patients Continue to Struggle for Years After Diagnosis
}

\author{
Emma Pennery, $C B E$
}

Clinical Director, Breast Cancer Care, London, UK

\begin{abstract}
One in six (17 \%) breast cancer patients calling Breast Cancer Care's Helpline in 2014 still needed information and support more than two years after diagnosis. This emphasises the necessity for ongoing care long after routine check-ups have ended.
\end{abstract}

\section{Keywords}

Breast cancer, patient care, supportive care

\begin{abstract}
Disclosure: Emma Pennery is an employee of Breast Cancer Care. No funding was received in the publication of this article.
Open Access: This article is published under the Creative Commons Attribution Noncommercial License, which permits any non-commercial use, distribution, adaptation and reproduction provided the original author(s) and source are given appropriate credit.

Received: 6 February 2015 Accepted: 6 February 2015 Citation: European Oncology \& Haematology, 2015;11(1):39-40 DOI: 10.17925/EOH.2015.11.01.39

Correspondence: Emma Pennery, Breast Cancer Care, 5-13 Great Suffolk Street, London SE1 ONS, UK. E: info@breastcancercare.org.uk
\end{abstract}

Every year, we receive nearly 12,000 calls to Breast Cancer Care's Helpline from people affected by breast cancer. Their reasons for calling encompass a broad range of topics, including concerns about symptoms or treatment side effects, making decisions about treatment and how to tell their children about their diagnosis.

But one noticeable theme is how long people continue to call after their diagnosis and after completion of treatment. Last year, one in six of our Helpline calls were from people still struggling with the aftermath of the disease and its treatment two or more years afterwards.

In some cases, people call even longer after diagnosis, and yet they still need support and questions answered. More than $8 \%$ of all our calls last year were from people who were five or more years past their diagnosis. ${ }^{2}$

Regular check-ups in the UK National Health Service (NHS) commonly stop at two to three years, and sometimes sooner with open access models of follow-up. However, the proportion of calls we receive so long after the end of hospital-based treatment emphasises what Breast Cancer Care has known for a long time: breast cancer does not end when treatment finishes; people continue to have ongoing information and support needs.

Those diagnosed two or more years before most commonly sought support for the following concerns:

- Debilitating side effects, including lymphoedema (swelling of the arm, hand or breast area) and hot flushes (17\%). ${ }^{3}$

- Emotional well-being, such as depression or lack of body confidence $(15 \%)^{4}$

- Concerns about ongoing treatments, such as tamoxifen (9\%). ${ }^{5}$

Also, in the years after hospital-based treatment, a vast majority are terrified the cancer will come back or are trying to adjust to a new normal after their life has been turned upside-down. Often they feel isolated, still struggling even as friends and family think it is time to celebrate the end of treatment.

One woman we spoke to, Helen, aged 45, a teacher from Torquay, UK, still experiences the effects of surgery four-and-a-half years after her diagnosis. She also suffers from severe fatigue that has degraded her quality of life enormously. She can no longer work full-time and struggles to visit friends or family. She continued to seek help and support from us despite having completed her hospital-based treatment more than two years previously.

She said: "Breast Cancer Care's Helpline has been a great support. They have answered any questions I had and gave me the reassurance and care I needed. I felt very empty and disorientated after treatment ended and it is comforting to know there is always somewhere to go with any concerns I may have."

We know that almost half of breast cancer patients feel that they are not given enough care and help from health or social services after leaving hospital subsequent to surgery. ${ }^{6}$ Today there are more than 550,000 people alive after a diagnosis of breast cancer in the UK; by 2020 , the figure is expected to reach 840,000 people. This growing population will continue to strain the capabilities of the NHS?

Also, with more than eight out of 10 people (85\%) surviving breast cancer for more than five years, the need for continued support and information is increasing. ${ }^{8}$ We need to ensure that patients' needs are met - but the demand on the NHS is becoming unmanageable.

We know the pressures on the NHS; we understand the difficulties. This is why support services, such as our Helpline, are so crucial. Referral to such services gives patients somewhere to turn as they deal with the ongoing effects of a breast cancer diagnosis. 


\section{Breast Cancer Editorial}

As more people are diagnosed and yet are also living longer with the disease, the Helpline offers valuable expert support from someone who understands, no matter the concern or question. We urge breast care nurses, oncologists and other healthcare professionals to refer patients to our service. There is help available - patients just need to know that it is there.

For support, call 08088006000 or visit www.breastcancercare.org.uk

Breast Cancer Care is the only specialist breast cancer support charity working throughout the UK. We were founded in 1973 by Betty Westgate, who was herself diagnosed with breast cancer. In the ensuing forty years, we have supported millions of women and their families through our face-to-face, phone and online services. We also provide training, support and networking opportunities to specialist breast cancer nurses, and Breast Cancer Care publications are used by the majority of breast cancer units throughout the UK. We campaign for better support and care and promote the importance of early detection, involving people with breast cancer in all that we do. Visit www.breastcancercare.org.uk or call our free Helpline at 08088006000.

1. Breast Cancer Care Helpline statistics report.17.4\% of people who called the Helpline in 2014 had been diagnosed two or more years before- 1,228 calls from a sample of 7,069 calls. Available at: https://www.breastcancercare.org.uk/ news/blog/breast-cancer-patients-still-struggling-years-afterdiagnosis (Accessed 1 April 2015).

2. Breast Cancer Care Helpline statistics report. People five or more years past diagnosis totalled 603 of $7,069(8.5 \%)$ Available at: https://www.breastcancercare.org.uk/news/ blog/breast-cancer-patients-still-struggling-years-afterdiagnosis (Accessed 1 April 2015).

3. Breast Cancer Care Helpline statistics report. 1,228 calls were from people diagnosed two or more years before. Of these calls, 204 (16.6\%) were assigned a call topic of "side effects-including lymphoedema, fatigue, hair loss and menopausal symptoms". Available at: https://www. breastcancercare.org uk/news/blog/breast-cancerpatients-still-struggling-years-after-diagnosis (Accessed 1 April 2015)
4. Breast Cancer Care Helpline statistics report. 1,228 calls were from people diagnosed two or more years before. Of these calls, 181 (14.7\%") were assign mo years before. Of these

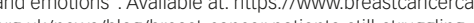
org.uk/news/blog/breast-cancer-patients-still-struggling-

5. Breast Cancer Care Helpline statistics. 1,228 calls were from people diagnosed two or more years before. Of these calls, 10 calls $(8.96 \%)$ were assigned a call topic of "treatment, all treatments other than surgery, including chemotherapy". Available at: https://www.breastcancercare.org.uk/news/ blog/breast-cancer-patients-still-struggling-years-afterdiagnosis (Accessed 1 April 2015)

6. Cancer Patient Experience Survey 2014. Figures show that "of those patients who said they needed it, 59 \% said they were definitely given enough care and help from health or social services after leaving hospital" . Available act: htps:// national-cancer-experience-survey/2014-nationat-cancer- patient-experience-survey/2014-national-cancer-patientexperience-survey-national-reports/688-2013-nationalcancer-patient-experience-survey-national-report-pdf/file, 98-9. Accessed 1 April 2015. The proportion of patients saying they were definitely given enough care and help from saying they were definitely given enough care and help from in the 2014 survey.

7. Maddams J, Utley M, Møller H, Projections of cancer prevalence in the United Kingdom, 2010-2040, Table 3: Complete prevalence in the United Kingdom, 2010-2040, by cance type and sex, under projection scenario 1, Br $J$ Cancer, 2012;107:1197.

8. Cancer Research UK. Figures are sourced from Cancer Research UK and refer to data from 2005 to 2009 and only apply to invasive breast cancer (not including DCIS). Availale at: http://info.cancerresearchuk.org/cancerstats/types/ breast/incidence/\#prev Accessed 1 April 2015. The tota number of calls answered by the Helpline during this period was 10,486 . 